\title{
Caracterización de las prácticas de innovación abierta en las pymes manufactureras en Bogotá
}

DOI: https://doi.org/10.21158/01208160.n90.2021.2931

Fecha de recepción: 03 de julio de 2020

Fecha de aprobación: 26 de marzo de 2021

Fecha de publicación: 25 de junio de 2021

\author{
María Alejandra Gómez-Cristancho ${ }^{1}$ \\ Universidad Distrital Francisco José de Caldas \\ maragomezc@correo.udistrital.edu.co \\ Laura Sofía Romero-Albarracín ${ }^{2}$ \\ Universidad Distrital Francisco José de Caldas \\ lsromeroa@correo.udistrital.edu.co \\ José Ignacio Palacios-Osma ${ }^{3}$ \\ Universidad Distrital Francisco José de Caldas \\ jpalacios@udistrital.edu.co
}

Cómo citar este artículo / To reference this article / Comment citer cet article / Para citar este artigo:

Gómez-Cristancho, M. A.; Romero-Albarracín, L. S.; Palacio2s-Osma, J. I. (2021). Caracterización de las prácticas de innovación abierta en las pymes manufactureras en Bogotá. Revista Escuela de Administración de Negocios, (90), 27-46.

DOI: https://doi.org/10.21158/01208160.n90.2021.2931

\section{Resumen}

En la actualidad, el principal objetivo de las organizaciones empresariales es aumentar la competitividad, por esta razón la innovación abierta se presenta como proceso facilitador en el desarrollo y el crecimiento de las empresas. Teniendo esto en cuenta, el objetivo del artículo es reconocer la disposición que existe por parte de las empresas a interactuar con otros actores del entorno, como, por ejemplo, la universidad, y así generar procesos colaborativos. Para esto se realizó un análisis de variables cualitativas a través de cuestionarios realizados a algunas empresas pymes — pequeñas y medianas empresas — del sector manufacturero de Bogotá y a docentes e investigadores de la Universidad Distrital. A partir de los resultados del estudio se formula un modelo de integración que caracteriza los factores claves para el desarrollo de procesos de innovación abierta entre los actores analizados. El modelo de integración propuesto busca resaltar los factores relevantes para los actores en estudio, con el fin de que, basados en este, sea posible definir claramente las metodologías, los acuerdos y las plataformas a utilizar entre las partes, garantizando la relevancia de los factores identificados y asegurando que no hay límite entre ellos en los procesos de innovación abierta. El análisis permite concluir que la implementación de este paradigma implica el establecimiento de relaciones de confianza, disposición de las partes, estructuración de un modelo de gestión de innovación y definición de los derechos de autor junto con acuerdos de cooperación claros y específicos.

Palabras clave: innovación; innovación abierta; modelo de innovación; gestión de innovación; sector manufacturero; pymes manufactureras; empresa y universidad.

1 Ingeniera Industrial - Universidad Distrital Francisco José de Caldas. ORCID: https://orcid.org/0000-0003-4448-1902

2 Ingeniera Industrial - Universidad Distrital Francisco José de Caldas. ORCID: https://orcid.org/0000-0003-3047-3418

3 Psicólogo - Universidad Santo Tomas. Administrador Hospitalario - Universidad Ean. Magister en E_learning y Redes Sociales UNIR. ORCID: https://orcid.org/0000-0003-4701-4373 


\title{
Characterization of open innovation practices in manufacturing SMEs in Bogotá
}

\begin{abstract}
Nowadays, the main objective of business organizations is to increase competitiveness, which is why open innovation appears as a facilitating process in business development and growth. With this in mind, the aim of this article is to recognize the willingness of companies to interact with other actors in the environment, for example universities, in order to generate collaborative processes. For this purpose, we carried out an analysis of qualitative variables by means of questionnaires to some SMEs -small and medium-sized enterprises- in the manufacturing sector in Bogotá, and to professors and researchers of Universidad Distrital. Based on the results, the study formulated an integration model that characterizes the key factors for the development of open innovation processes among the actors that were analyzed. The integration model proposed seeks to highlight the factors that may be relevant for the actors under study, so that, it is possible to clearly define the methodologies, agreements, and platforms to be used between the parties, guaranteeing the importance of the factors that were identified and assuring that there is no limit between them in the open innovation processes. The analysis allows to conclude that the implementation of this paradigm implies the establishment of trusting relationships, the willingness of the parties, the structuring of an innovation management model, and the definition of copyrights together with clear and specific cooperation agreements.
\end{abstract}

Keywords: innovation; open innovation; innovation model; innovation management; manufacturing sector; manufacturing SMEs; business and university.

\section{Caracterização das práticas de inovação aberta em PMEs manufatureiras em Bogotá}

\section{Resumo}

Atualmente, o principal objetivo das organizações empresariais é aumentar a competitividade, por isso a inovação aberta se apresenta como um processo facilitador no desenvolvimento e crescimento das empresas. Diante disso, o objetivo do artigo é reconhecer a disposição que existe por parte das empresas de interagir com outros atores em sua volta, como, por exemplo, a universidade, e assim gerar processos colaborativos. Para isso, foi realizada uma análise de variáveis qualitativas por meio de questionários aplicados a algumas PMEs - pequenas e médias empresas - do setor manufatureiro de Bogotá e a professores e pesquisadores da Universidade Distrital. Com base nos resultados do estudo, é formulado um modelo de integração que caracteriza os fatores-chave para o desenvolvimento de processos de inovação aberta entre os atores analisados. $\mathrm{O}$ modelo de integração proposto busca evidenciar os fatores relevantes para os atores em estudo, para que, a partir disso, seja possível definir com clareza as metodologias, acordos e plataformas a serem utilizadas entre as partes, garantindo a relevância dos fatores identificados. e garantindo que não haja limite entre eles nos processos de inovação aberta. A análise permite concluir que a implementação deste paradigma implica o estabelecimento de relações de confiança, disposição das partes, estruturação de um modelo de gestão da inovação e definição de direitos de autor juntamente com acordos de cooperação claros e específicos.

Palavras-chave: inovação; inovação aberta; modelo de inovação; gestão da inovação; setor manufatureiro; PMEs manufatureiras; empresa e universidade. 


\section{Caractérisation des pratiques d'innovation ouverte dans les manufactures de Bogotá}

\section{Résumé}

L'un des principaux objectifs des entreprises est d'améliorer leur compétitivité au travers de l'innovation pour permettre le développement et la croissance de leurs affaires. L'objectif de cet article est d'analyser la disposition des entreprises à interagir avec d'autres acteurs de leur environnement, comme les universités, pour générer des processus collaboratifs. Une analyse de variables qualitatives a ainsi été réalisée au travers de questionnaires adressés à certaines PME - petites et moyennes entreprises - du secteur manufacturier de Bogotá et à des enseignants et chercheurs de l'Université Distrital. Les résultats de l'étude permettent de formuler un modèle d'intégration définissant les facteurs clés pour le développement des processus d'innovation. Le modèle d'intégration proposé met en évidence les facteurs pertinents pour les acteurs étudiés afin de définir clairement les méthodologies, les accords ou les plateformes à utiliser entre les parties pour garantir la pertinence des facteurs identifiés, et s'assurer qu'il n'existe pas de limitation dans les processus d'innovation ouverte. L'analyse conclut que la mise en œuvre de ce paradigme implique l'établissement de relations de confiance, la disposition des parties, la structuration d'un modèle de gestion de l'innovation et la définition du droit d'auteur et d'accords de coopération clairs et spécifiques.

Mots-clés: innovation; innovation ouverte; modèle d'innovation; gestion de l'innovation; secteur manufacturier; PME manufacturières; entreprise et université. 


\section{Introducción}

$\mathrm{L}$ a innovación se ha convertido en un factor sustancial para aumentar la competitividad en las organizaciones debido a los entornos VUCA -Volatility, Uncertatinty, Complexity, Ambiguity - que demandan flexibilidad y rapidez de respuesta por las empresas, a fin de mantener su competitividad (Glukhova et al., 2020). En tal sentido, la consolidación de relaciones de interdependencia entre diferentes actores de la sociedad y la economía introduce el concepto de innovación abierta como un modelo de cocreación y cooperación que permite implementar nuevas ideas, productos y procesos de manera integral y con apoyo de recursos de diferentes organizaciones (Muhammad, Ikram, Jafri y Naveed, 2021).

El modelo de innovación abierta plantea que la empresa debe identificar e incorporar ideas y tecnologías externas a la organización, intercambiar con terceros las tecnologías o los conocimientos desarrollados en el interiory desarrollar innovaciones de forma colaborativa con alguno o todos los actores de los modelos de hélice - triple, cuádruple o penta hélice- (Cabrera-Flores et al., 2020; GarcíaGonzález y Ramírez-Montoya, 2019).

Al revisar el horizonte de posibles actores participantes en procesos de innovación abierta se reconoce el papel de las instituciones de educación superior (IES) como un potencial motor de generación de conocimiento (Zakharov, Posazhennikov y Zakharova, 2020). De acuerdo con los estudios realizados y las experiencias documentadas, se evidencia el rol que puede asumir la educación superior frente a los procesos de innovación, en particular por la disponibilidad tanto de talento humano como de su capacidad de absorción de tecnologías y conocimientos (JucaAulestia et al., 2020).
Los modelos de hélice (Crilly et al., 2020) requieren que se defina un actor central responsable de integrar y coordinar las capacidades de innovación tanto de la misma institución como de las capacidades de los demás integrantes, de modo que así se alcanzan beneficios para todas las partes, respetando su autonomía y gestión de conocimiento. Estas alianzas o estrategias de cocreación llevan a alcanzar sinergias en el proceso de innovación, con lo cual se obtiene una reducción de costos y riesgos con beneficios para los involucrados.

Así, el objetivo de la investigación consistió en identificar las prácticas y la disposición de desarrollar procesos de innovación abierta en las pymes manufactureras y la integración con el sector académico, en espera de que los resultados de este estudio contribuyan a la implementación y el reconocimiento de las pymes frente a este paradigma de innovación.

El artículo se desarrolla de la siguiente forma: primero, se define el concepto de innovación abierta; segundo, se presenta un análisis de la innovación en el contexto empresa y universidad; tercero, se describe la metodología desarrollada; cuarto, se presentan los resultados obtenidos, que incluyen un análisis de correlación de factores cualitativos influyentes para cada actor - empresauniversidad - en el proceso de innovación abierta, y por último, se presenta la propuesta de un modelo de integración para la implementación de la innovación abierta en el binomio universidadpyme a partir de la revisión bibliográfica y los resultados de la investigación. 


\section{Marco teórico}

$\mathrm{C}$ hesbrough (2003) introdujo el concepto de innovación abierta como un paradigma que asume que las empresas pueden y deben usar ideas, conocimientos y tecnologías externas como un modelo de colaboración y transferencia tecnológica en el que los involucrados puedan reducir los riesgos de la innovación y a la vez tener acceso al recurso y al talento humano de otras organizaciones, a fin de obtener beneficios para todos los participantes. La innovación abierta combina ideas internas y externas en arquitecturas y sistemas cuyos requisitos están definidos por un modelo de negocio, lo cual permite lograr mejores resultados y aprovechar las capacidades que pueden aportar actores externos, flexibilizando los límites de las organizaciones y mejorando sus capacidades dinámicas y de innovación (Yuan, Vlas y Peng, 2018).

Por otra parte, Huizingh (2011) y Shutyak (2016) consideran la innovación abierta como el proceso por medio de cual los conocimientos fluyen entre el entorno interno y externo de las empresas, de manera que generan entradas y salidas que fortalecen la generación del flujo de ideas, lo que resulta en el aumento de la innovación interna junto con la expansión de las redes externas que permiten aumentar las opciones de obtener innovación de otros entes. Además, establecen que la innovación abierta integra la innovación saliente al ofrecer un marco en el que cualquier producto intermedio de procesos de innovación se considera un bien económico que puede ser explotado internamente y/o externamente por otros aliados.

El concepto de innovación abierta ha sido abordado por muchos autores, y su aplicación depende de la perspectiva desde la que se considere, pasando desde un enfoque orientado al mercado hasta enfoques centrados en las capacidades de la organización. Sin embargo, a partir de la revisión bibliográfica se hace relevante resaltar que se debe contar con un stakeholder principal o líder que asuma la gestión del proceso, el cual puede ser la universidad, la cual, dadas sus condiciones, puede definir políticas y lineamientos para la cocreación y el establecimiento de diversas estrategias de colaboración (Figura 1).

Figura 1. Factores de gestión para innovación abierta

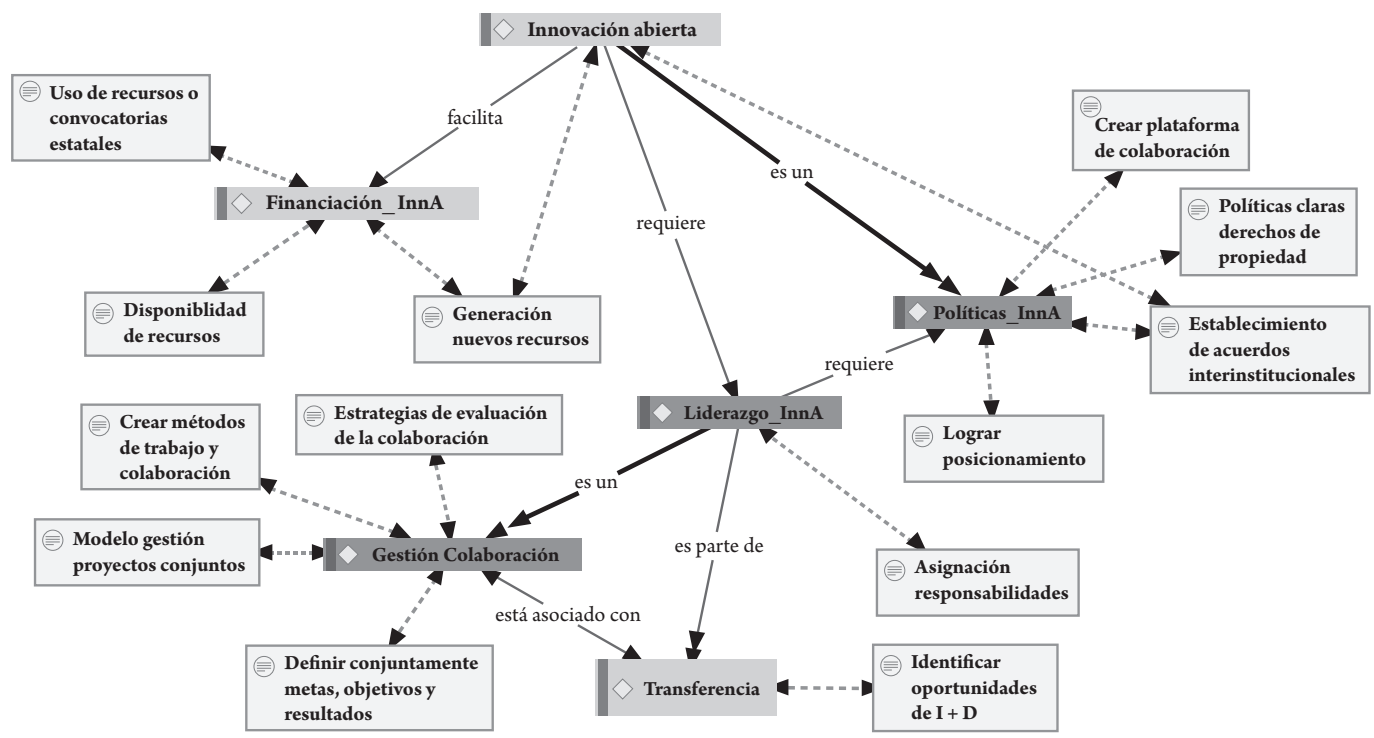

Fuente. Elaboración propia a partir de revisión bibliográfica. 
Así, por ejemplo, la innovación es un aspecto clave para la sostenibilidad y la competitividad de las organizaciones, y de la economía, en general, así como una estrategia dirigida a responder a los Objetivos de Desarrollo Sostenible (Castellar, 2020). Por otra parte, en los últimos años la innovación abierta ha incursionado en modelos de negocios abiertos en los que las fuentes y el intercambio de conocimientos se extienden más allá de las fronteras de la organización (de las Heras-
Rosas y Herrera, 2021). Así mismo, se comprende que en el interior de las empresas no siempre se cuenta con todos los recursos y conocimientos necesarios para enfrentar los entornos VUCA.

Adicionalmente, en conformidad con la experiencia, en diferentes sectores se han definido y enfocado diversas formas de compresión de la innovación abierta (véase la Tabla 1).

Tabla 1. Algunos conceptos de innovación abierta

\begin{tabular}{|l|l|}
\hline \multicolumn{1}{|c|}{ Autor } & \multicolumn{1}{c|}{ Definición } \\
\hline Ovuakporie et al. (2021) & $\begin{array}{l}\text { Aprovechar el conocimiento ampliamente disperso de múltiples organizaciones, } \\
\text { lo cual permite adquirir recursos complementarios, compartir costos y riesgos } \\
\text { asociados con I + D. }\end{array}$ \\
\hline Payán-Sánchez et al. (2021) & $\begin{array}{l}\text { Campo de investigación reconocido y complejo con múltiples facetas, debido al } \\
\text { proceso inherentemente dinámico que conlleva colaboración para tener acceso a } \\
\text { nuevos conocimientos y recursos adicionales. }\end{array}$ \\
\hline Yun et al. (2020) & $\begin{array}{l}\text { Enfoque holístico que explora conscientemente las capacidades y los recursos } \\
\text { de fuentes internas y externas frente a diversas oportunidades que se divulgan y } \\
\text { comercializan por múltiples canales. }\end{array}$ \\
\hline $\begin{array}{l}\text { Genuchten, Calderón- } \\
\text { González y Mulder (2019) }\end{array}$ & $\begin{array}{l}\text { La aceleración de los procesos de innovación interna de una organización mediante el } \\
\text { uso deliberado de conocimiento externo y la salida del conocimiento interno. }\end{array}$ \\
\hline $\begin{array}{l}\text { García-González y Ramírez- } \\
\text { Montoya (2019) }\end{array}$ & $\begin{array}{l}\text { Se caracteriza por la libre transferencia de conocimiento entre los diferentes actores } \\
\text { involucrados, tales como universidades, empresas y gobierno. Es un nuevo imperativo } \\
\text { para crear y aprovechar la tecnología, por el cual una organización puede mejorar sus } \\
\text { capacidades de innovación mediante la interacción con otras organizaciones. }\end{array}$ \\
\hline Chesbrough (2003) & $\begin{array}{l}\text { Fenómeno en el que las empresas hacen un mayor uso de las ideas y tecnologías } \\
\text { externas en su propio negocio, además, dejan que las ideas y tecnologías internas que } \\
\text { no se utilizan salgan para que otros las usen en sus negocios. }\end{array}$ \\
\hline
\end{tabular}

Fuente. Elaboración propia.

Es así como la innovación abierta redefine las fronteras de la organización, de modo que al cuestionar los modelos de negocio cerrados se reconoce que existe una diversidad de actores externos que pueden coadyuvar en el desarrollo de mejores procesos de innovación. Así, la innovación implica la consolidación de redes colaborativas definidas como un equipo de personas automotivadas (Lam, Nguyen y Tran, 2021; Wu, Garimella y Subramanyam, 2021), con una visión colectiva para colaborar en alcanzar un objetivo común a través del intercambio de ideas, información y trabajo, de manera tal que las redes colaborativas permiten que los equipos compuestos por actores internos y externos trabajen en el desarrollo de una única idea. Esta facilita que los proyectos resultantes entren y salgan en varios puntos y de distintas formas, lo que arroja como resultado varias vías por las cuales pueden salir productos al mercado (Flor, Blasco-Díaz y LaraOrtiz, 2020; Gloor, 2006). 
De esta manera, la innovación no se focaliza exclusivamente en el nivel interno de una compañía, sino que busca diversificar sus opciones en recursos, emprendimientos, licencias y alianzas con terceros, sean estos empresas, usuarios o proveedores, entre otros, con lo que se amplía así la búsqueda de información, los conocimientos y la tecnología para fortalecer la innovación. También de esta forma se centra en una diversidad de fuentes de recurso de financiación _crowdfunding-, de comercialización y de trabajo colaborativo crowdsourcing - para la generación de nuevos productos, servicios y modelos de negocio (Temiz, 2021; Yun y Liu, 2019).

Varios autores hacen un llamado a la importancia de estudiar más a profundidad las redes, las estrategias, los clientes, el marketing y la cocreación, elementos que facilitan los modelos de innovación abierta, considerando que si bien existe una caracterización de esta, se requiere aún indagar sobre los mecanismos tanto internos como externos que facilitan estas alianzas de colaboración y cooperación en el intercambio de ideas y capacidades, así como mostrar la importancia de investigar el entorno como un todo y no con actores individuales (Becker y Eube, 2018; Zobel, Lokshin y Hagedoorn, 2017).

De esta manera, la innovación abierta genera una apertura holística y los empresarios inmersos en esta práctica, que toman decisiones a partir de diversas opciones y recursos, son conscientes de todas las posibilidades que pueden ser aprovechadas $y$ de cómo hacerlo de forma efectiva, junto con la participación de actores externos (Valdez-Juárez y Castillo-Vergara, 2020).

\subsection{Innovación abierta: contexto empresa y universidad}

Las empresas sufren los efectos macroeconómicos del entorno mundial y los requerimientos del cliente que cambian constantemente, por ende, deben invertir en innovación de producto, proceso y servicio. Por esta razón, la investigación, el desarrollo y la innovación $-\mathrm{I}+\mathrm{D}+\mathrm{i}-$ debe ser vista bajo una perspectiva colaborativa, con capacidad de compartir con otras organizaciones o actores de los modelos de hélice, lo cual permitiría optimizar, disminuir y aprovechar los recursos o conocimientos de estas para respaldarse entre sí, cocrear y así posicionarse en el mercado nacional e internacional (Liu, Du, Hong, Fan y Wu, 2020; López-Garzón y Vaca-Sánchez, 2010). De igual forma, evitar asumir el desarrollo de manera aislada en la medida en que no siempre se tiene todo lo necesario para mejorar su competitividad.

En este sentido, se hace ostensible que las organizaciones comiencen a trabajar de forma colaborativa consolidando una cultura de innovación abierta (Vlajcic, Marzi, Caputo y Dabic, 2019) para generar innovaciones flexibles, mediante la creación de acciones de alto impacto que permitan el aumento de la rentabilidad, la reducción de costos y la optimización de recursos; es necesario que las empresas aprovechen sus departamentos $\mathrm{I}+\mathrm{D}$ y exploren con otras organizaciones el desarrollo y la comercialización de productos (Bernal-Torres y Frost-González, 2015; Vicente-Saez, Gustafsson, Van den Brande, 2020).

Progresivamente, la innovación abierta en Colombia es una práctica que está en crecimiento, se estudia desde el ámbito académico y busca alcanzar una adecuada validación práctica. Programas como INNpulsa (2021) lidera diversos proyectos nacionales de innovación abierta y logra acuerdos colaborativos entre empresas como Ecopetrol, Argos, Tipiel, Servientrega, Belcorp, Aldor, Cotecmar y Conconcreto con otros actores -empresas, investigadores, universidades-. También ha facilitado la creación de acuerdos entre entidades públicas de la talla del Instituto Nacional de Vigilancia de Medicamentos y Alimentos (Invima), la Unidad de Restitución de Tierras y gobernaciones como las de Cundinamarca y el Valle del Cauca. 
Así mismo, empresas como Ecopetrol desarrollan desde 2019 proyectos de innovación abierta con alianzas estratégicas con actores del ecosistema de ciencia y tecnología, con programas tales como Innóvate, C-Emprende, Ruta N, Plug and Play y Hackatons (Ecopetrol, 2019).

A nivel nacional, las pymes también desarrollan innovación. Según la Encuesta de Desarrollo e Innovación Tecnológica (EDIT) realizada por el Departamento Administrativo Nacional de Estadística (DANE, 2019), se encontró que «11 empresas fueron innovadoras en sentido estricto, 1.561 empresas fueron innovadoras en sentido amplio, 278 empresas fueron potencialmente innovadoras y las 5.679 empresas restantes no fueron innovadoras» (p. 5). Esto evidencia la importancia que fortalecer la cultura $y \ll l a$ innovación conduce al crecimiento económico, más empleo, mayores ingresos, mejoras en la calidad de vida de la población y competitividad de los países al tener un impacto significativo en los niveles de productividad» (p. 5).

Aunque los directivos tienen en sus planes a largo plazo aumentar la innovación en las empresas, según los antecedentes descritos en la encuesta hecha por la Asociación Nacional de Instituciones Financieras (ANIF, 2020), se evidencia una falta de conciencia sobre la importancia de innovar, pues se otorga más valor a otras acciones de mejoramiento tales como a) capacitar a su personal, b) obtener una certificación de calidad y c) lanzar un nuevo producto.

Por otra parte, las instituciones de educación superior, si bien reconocen como sus funciones sustantivas la docencia, la investigación y la extensión, en los últimos años se han interesado - en el caso de Colombia - en tener un mayor impacto en la sociedad y en su entorno. En este sentido, se conforman, según Colciencias (2021), las Oficinas de Transferencia de Resultados de Investigación (OTRIS), que tienen como misión conectar la demanda de tecnologías - necesidades y retos de las empresas- con la oferta — producción de las universidades y centros generadores de conocimiento-.

De esta forma, las universidades se visibilizan cada vez más como organizaciones generadoras de conocimientos y tecnologías, no solo para sus propósitos de ranquin institucional y de calidad, sino como un actor propulsor de desarrollo económico e innovación para la industria, la sociedad y el Gobierno (Sudiana, Sule, Soemaryani y Yunizar, 2020). Así, la universidad se desarrolla de manera ambidiestra, tanto desde lo académico como desde la innovación y el emprendimiento (Palacios, Ramírez-Montoya, Cubo-Villalba y Burgos, 2018).

De esta manera, el paradigma de innovación abierta para la universidad implica un compromiso con establecer estrategias y mecanismos de integración y cooperación con diferentes sectores de la sociedad y la economía, así como con direccionar parte de sus esfuerzos a proyectarse como organización en comercializar e intercambiar tecnologías y conocimientos, y ofrecer soluciones a problemáticas sociales y ambientales, estableciendo relaciones de cocreación con gran capacidad de absorción e innovación a la luz de los retos de la sociedad (Hasche, Linton y Oberg, 2017).

Así mismo, la gestión del conocimiento se presenta como un valor intangible para el desarrollo de la innovación, y la universidad, como principal generadora de conocimiento, es importante para la transferencia de este (Jamett, Alvarado y Maturana, 2017). No obstante, gran parte de las empresas no tienen conexiones con las universidades y asumen sus retos como una organización cerrada; así mismo, se evidencia que en la labor investigativa empresarial solo en casos esporádicos se motiva e incentiva a los investigadores empresariales (González-Millán, Rodríguez-Díaz y GonzálezMillán, 2019). 


\section{Metodología}

B asados en la revisión bibliográfica y con el fin de dar cumplimiento al propósito de la investigación, así como para confrontar el modelo del paradigma de innovación abierta frente a la práctica de esta en algunas empresas colombianas, se toma una muestra de industrias manufactureras correspondiente a la división 10 y 33 del código CIIU —clasificación industrial internacional uniforme- en la sección C, clasificación dada por la Dirección de Impuestos y Aduanas Nacionales (DIAN) como la actividad económica. La recolección de datos se obtuvo de la participación voluntaria de 25 empresas pymes manufactureras de Bogotá.

El cuestionario cuenta con preguntas de carácter cualitativo, lo cual permite realizar análisis de comportamientos intangibles que pueden tener una alta relevancia en la propuesta a realizar en esta investigación. Se midieron aspectos tales como importancia de la innovación, los procesos de innovación y las prácticas de innovación abierta e interés en la cocreación.

Por otra parte, se aplicó un cuestionario electrónico a 78 docentes de la Universidad Distrital Francisco José de Caldas, a fin de conocer e identificar su interés en desarrollar proyectos de $\mathrm{I}+\mathrm{D}+\mathrm{i}$-investigación, desarrollo e innovación - con el sector productivo que permitan realizar transferencia tecnológica y de conocimiento, en el contexto del paradigma de la innovación abierta. En la selección de los docentes participantes se usó el modelo de Barroso y Cabero (2013) para identificar el nivel coeficiente de competencia - K-, con el propósito de garantizar el conocimiento del experto en el tema de investigación e innovación, a partir de la siguiente formulación:

$$
K=\frac{1}{2\left(K_{\mathrm{c}}+K_{a}\right)}
$$

Donde:

- $K$ : es el «coeficiente de conocimiento»e información que tiene el experto acerca de la innovación abierta. Se calcula a partir de la valoración que realiza el propio experto en la escala del 0 al 10, multiplicado por 0,1 .

- $K_{a}$ : es el denominado «coeficiente de argumentación» o fundamentación de los criterios de los expertos. Este coeficiente se obtiene a partir de la asignación de una serie de puntuaciones a las distintas fuentes de argumentación que ha podido obtener del experto.

Con los siguientes criterios de selección:

- Si $0,8<K<1,0$ coeficiente de competencia experta alto $=28$ docentes.

- Si $0,5<K<0,8$ coeficiente de competencia experta medio $=30$ docentes.

- Si $K<0,5$ coeficiente de competencia experta bajo $=20$ docentes.

Quedan así habilitados, finalmente, 58 docentes, a quienes se les aplicó el cuestionario respectivo. Cabe aclarar que los dos grupos muestrales respondieron en torno a actores generales, es decir, los encuestados respondieron las preguntas sin referirse a algún actor en particular.

Cabe señalar que esta investigación estudia las prácticas de innovación abierta en algunas pymes manufactureras en Bogotá en el marco temporal de los años 2019 y 2020 como parte del estudio realizado en el grupo de investigación Gicoecol de la Universidad Distrital Francisco José de Caldas, con el fin de determinar la disposición de los actores empresa-universidad para realizar procesos de innovación colaborativa. 


\section{Resultados}

\subsection{Resultados-empresas}

A continuación, se presenta el análisis por secciones del cuestionario realizado a las empresas.

\subsubsection{Importancia de la innovación.}

Con relación a las preguntas sobre la innovación, se identificó que el $88 \%$ de las empresas consideran la innovación un factor diferencial para la aumentar la competitividad, y solo el $12 \%$ no la considera y no aplican procesos de innovación, pero sí conocen el concepto.

Así mismo, durante los años 2018 y 2020 el 80 \% de las empresas introdujo algún tipo de innovación, principalmente nuevas o de modificaciones a productos y procesos. No existe la tendencia a hacer innovación en modificaciones a la estructura organizacional, lo que puede significar falta de agilidad en el interior de las organizaciones para responder a comportamientos emergentes. Por otra parte, las empresas que han introducido algún tipo de innovación recientemente lo han hecho con la innovación tradicional y son pocas las que han hecho algún tipo de innovación con otros actores - universidad o empresa-. De esto se infiere que las empresas estudiadas no confían en los nuevos paradigmas de innovación o no tienen conocimiento sobre cómo desarrollarlos.

\subsubsection{Inversión en innovación.}

Al indagar sobre la financiación para realizar innovación, más de la mitad de las empresas consideran estos procesos una inversión con retorno a largo plazo, por lo cual resulta evidente recurrir a financiación (Figura 2).

Figura 2. Papel de la financiación para realizar innovación en su empresa

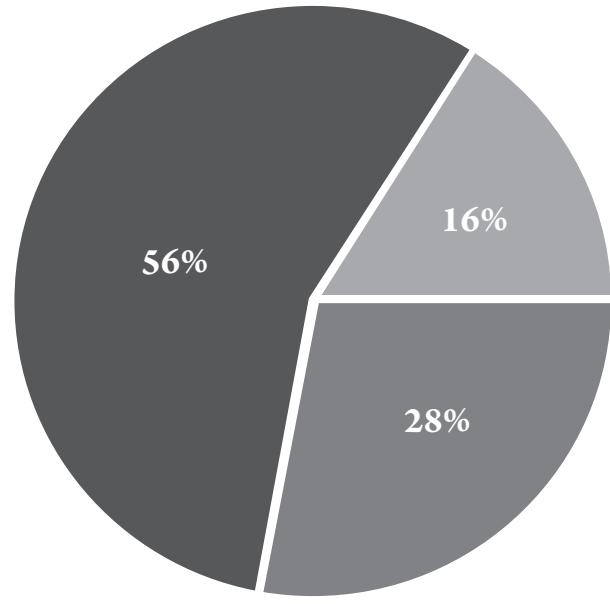

Fuente. Elaboración propia.

En lo que se refiere a las empresas que no han introducido algún tipo de innovación, sus razones son organizacionales, en lugar de falta de financiación. Las empresas incurren en financiación externa, especialmente a préstamos, de manera que una de las principales dificultades que encuentran son las tasas de interés, según el informe de la ANIF. A pesar de que conocen la existencia de financiación pública no se apoyan en ella por falta de conocimiento sobre los tipos existentes y 
sobre cómo acceder, o por incumplimiento de los requisitos necesarios para postularse; además, les es más fácil pedir créditos. Los recursos asignados a través de Colciencias que apoyan procesos de innovación en las empresas no se utilizan porque los desconocen, o porque se presentan proyectos que no son financiables.

Dentro de las empresas, al incentivar la innovación tienden a hacer reuniones para la propuesta de nuevas ideas y de prototipos de los productos. Aun así, algunas de las organizaciones tienden a innovar solo cuando la alta dirección ve la necesidad y no se desarrollan procesos de innovación de forma constante.

Con respecto a la rapidez de las empresas para realizar cambios, estas reconocen que poseen un alto grado (Figura 3), lo cual evidencia niveles importantes relacionados con flexibilidad y capacidad de absorción de nuevos conocimientos e información. Téngase en cuenta que 1 es poca rapidez y 7 es mayor rapidez.

Figura 3. Rapidez de la organización para realizar cambios

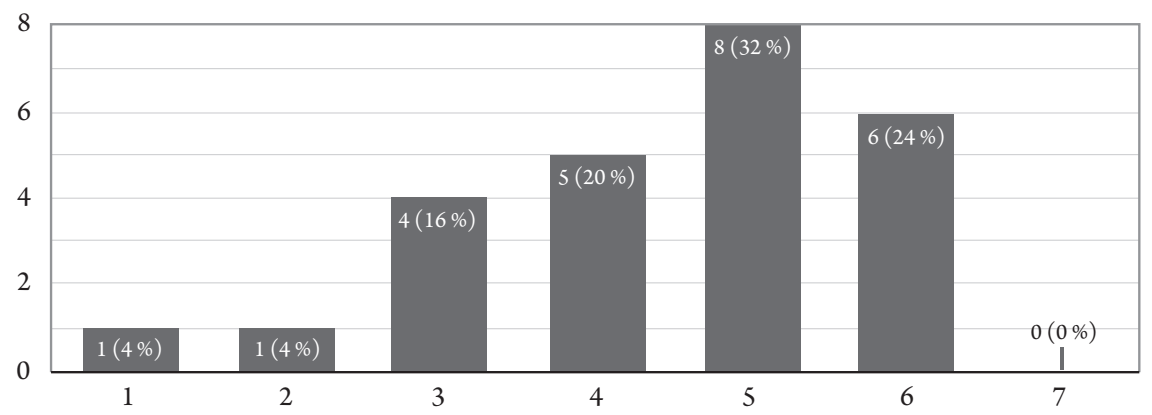

Fuente. Elaboración propia.

La mayoría de las empresas recogen datos sobre sus innovaciones, pero el $36 \%$ no los analiza para tomar acciones en sus innovaciones futuras o no los recoge, y el $48 \%$ no retroalimenta sus innovaciones, por lo que no toman acciones de sus lecciones aprendidas.

\subsubsection{Participación en innovación abierta.}

Con respecto a la innovación abierta se evidencia que el $40 \%$ de las empresas han escuchado el concepto de innovación abierta y para otras es totalmente nuevo; así, el $60 \%$ de las empresas no conocen en qué consiste ni cómo se define la innovación abierta.

Teniendo en cuenta el concepto de innovación abierta como una estrategia que permite el trabajo cooperativo con otros actores, en el que se combinan los conocimientos y las ideas de todos para crear nuevos desarrollos, el $32 \%$ de las empresas encuestadas no consideran trabajar con otros actores.

Actualmente, el $64 \%$ de las empresas tienen innovaciones en curso, pero lo hacen de manera interna y la disposición a trabajar con otros actores es baja a causa de la falta de confianza, ya que consideran riesgoso desarrollar innovaciones en conjunto con otras organizaciones, especialmente con empresas que pueden significar competencia, tal como lo expresan en una de las respuestas de los participantes: «Por qué en Colombia no existe el secreto industrial y no estamos protegidos en temas de innovación. Todo el mundo puede copiar las innovaciones así se firmen compromisos de reserva. No existe ética en este país». 
Así mismo, se evidencia que las empresas tienen dificultad para visualizar las ventajas que trae la innovación abierta, dado que sus pares en el mercado no la practican e igualmente tienen buenos resultados en competitividad. Sin embargo, reciben ideas tanto internas como externas a pesar de no trabajarlas conjuntamente; el $36 \%$ de las empresas han participado en retos de innovación trabajando con otros actores y reportan una buena experiencia, mientras el $56 \%$ ha pensado en participar, pero no lo ha hecho por miedo a perder recursos estratégicos por propiedad intelectual.

Aun así, el $84 \%$ está dispuesto a trabajar con la universidad, del cual el $32 \%$ ya lo hecho una o dos veces al año y con innovaciones puntuales a fin de trabajar con otras empresas, y solo el $28 \%$ no está dispuesto a hacerlo.

Uno de los resultados más importantes es que la estructura organizacional que tienen actualmente las empresas no les facilita generar procesos de innovación abierta. El $88 \%$ de las empresas encuestas mencionan que si cambiaran su estructura organizacional estarían dispuestos a cambiar decisiones estratégicas relacionadas con objetivos y políticas para implementarla, ya que consideran que es posible mejorar la productividad y la agilidad en los procesos de innovación con soluciones integrales, pero aún existe el miedo a perder conocimiento y tiempo.

Solo el $12 \%$ de las empresas no están dispuestas a cambiar sus políticas y los objetivos, debido a que los consideran adecuados; si los cambiaran tendrían que abandonar la estrategia que han construido a lo largo del tiempo y perder así la columna vertebral de la empresa.

\subsection{Resultados-docentes}

A continuación, se analizan los resultados obtenidos de la encuesta realizada a 58 docentes e investigadores de la Universidad Distrital Francisco José de Caldas.

\subsubsection{Experiencia y nivel de argumentación.}

Se observa en la figura 4 que, en promedio, los encuestados califican en 5,9 sus conocimientos, su experiencia o participación en procesos de transferencia tecnológica, intercambio de conocimiento e innovación abierta. Esto quiere decir que su posible participación en contextos relacionados con innovación abierta es medianamente activa y claramente no es nula.

Figura 4. Resultados de investigadores y docentes según nivel de conocimiento

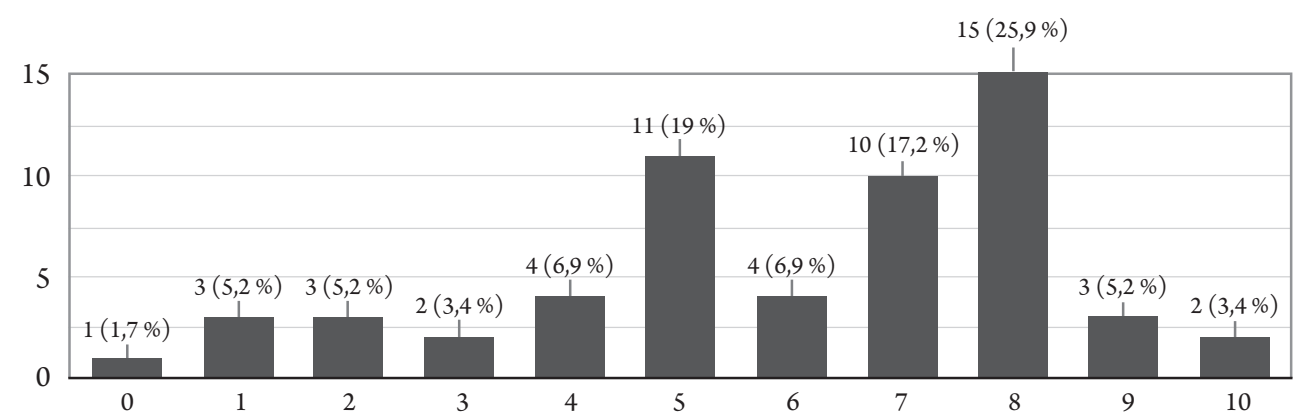

Fuente. Elaboración propia.

Conforme a los resultados, se puede inferir que el $43 \%$ de los encuestados califica como media su experiencia obtenida participando en proyectos relacionados con la innovación abierta y la transferencia de conocimientos mediante análisis teóricos, investigaciones, desarrollos y aplicaciones. Se observa que 
solo un tercio de los encuestados calificó la experiencia obtenida desarrollando actividades relacionadas como alta, lo cual quiere decir que aún hace falta introducir a los docentes e investigadores en contextos de innovación abierta (Figura 5).

Figura 5. Resultados de investigadores y docentes según el nivel de argumentación

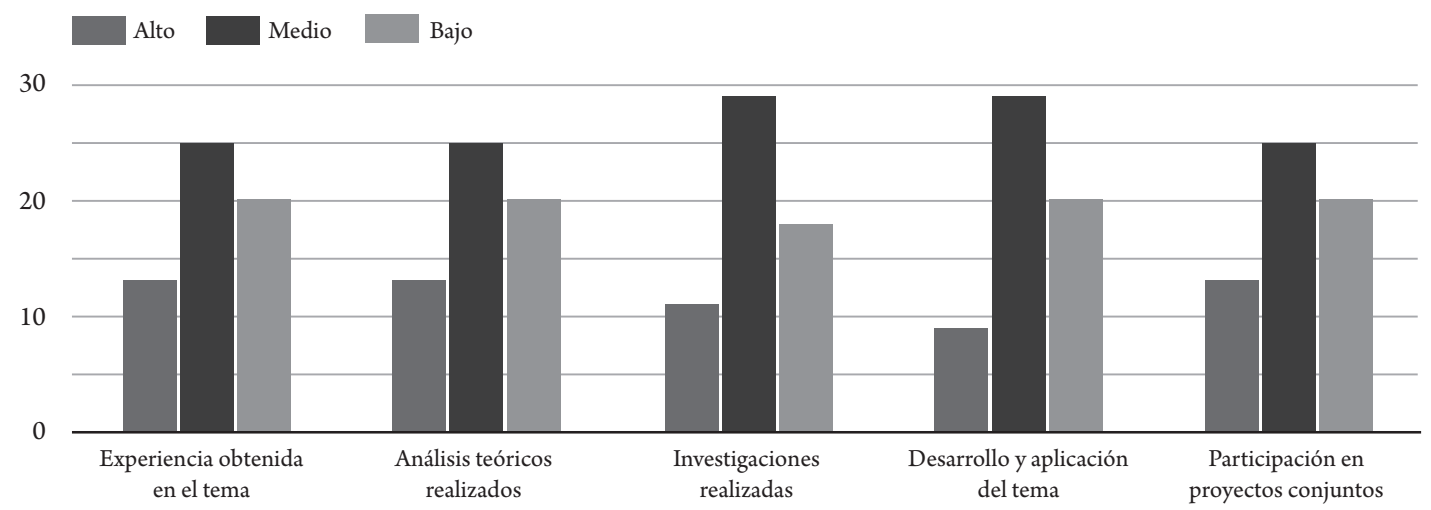

Fuente. Elaboración propia.

El $56,8 \%$ de los encuestados expresa que la Universidad Distrital cuenta con mecanismos que permiten establecer o desarrollar proyectos de investigación e innovación con otras universidades de forma frecuente o muy frecuente. De igual forma, el mismo porcentaje de encuestados asegura que la universidad cuenta o permite establecer convenios para desarrollar proyectos que busquen solucionar necesidades en el sector público.

En comparación con el establecimiento del sector privado, en cuanto a la calificación según la cual la Universidad Distrital genera entornos para la realización de proyectos de investigación e innovación conjunta, el 44,8 \% de los encuestados considera que la universidad lo permite frecuente o muy frecuentemente, y un $27,5 \%$ de los encuestados considera que la universidad lo permite ocasionalmente.

Casi un tercio de los encuestados considera que la Universidad Distrital no fomenta el intercambio de ideas, conocimientos y tecnologías entre académicos, sector productivo y sector social. Se puede evidenciar una oportunidad por parte de la Universidad Distrital como figura motora de conocimiento para la sociedad, al integrarla relación universidad-empresa-Estado, según se propone en la triple hélice de Sabato y Botana (1993), o en la triple hélice de Etzkowitz (2003).

Esto se apoya en la perspectiva dividida de los encuestados con respecto a las fuentes de financiación con las que cuenta la Universidad Distrital para apoyar el desarrollo de proyectos de investigación e innovación. A pesar de las diferentes consideraciones con respecto a las fuentes de financiamiento, un total de 25 encuestados considera que la universidad frecuenta la vinculación con diferentes sectores para el desarrollo de proyectos.

El enfoque investigativo de la Universidad Distrital está alineado con la perspectiva de sus docentes e investigadores, debido a que más de la mitad de los encuestados considera que la cultura de investigación e innovación de la universidad cuenta con docentes, estudiantes e investigadores emprendedores. Además, muy de la mano se percibe un deseo de participación en la solución de problemas reales, sociales o productivos. $\mathrm{Al}$ ser una universidad de carácter público, los procesos 
de innovación e investigación que se realizan tratan siempre de tener en cuenta que sus resultados tengan un amplio impacto en el país y en la sociedad en general.

\subsubsection{Interés de los docentes.}

El $63,7 \%$ de los docentes e investigadores encuestados de la Universidad Distrital está interesado en compartir conocimiento con otros sectores o instituciones. Sin embargo, consideran que se les incentiva de forma ocasional a participar en proyectos de transferencia tecnológica y de conocimiento. Por otro lado, un poco menos de la mitad considera que se cuenta con estrategias y mecanismos legales para proteger los conocimientos y tecnologías que se usen o creen en proyectos con investigadores externos.
Tan solo un 5,1\% de los encuestados no estaría dispuesto a desarrollar innovación e investigación con otras entidades públicas. El mismo porcentaje no está dispuesto a desarrollar innovaciones e investigación con otras instituciones privadas. Más del $70 \%$ de los encuestados considera pertinente que la Universidad Distrital comercialice los resultados de las investigaciones o innovaciones desarrolladas.

Solo nueve de los docentes e investigadores no están de acuerdo con que los procesos de investigación o innovación serían más efectivos si se desarrollan con otras instituciones. Según la figura 6 , se evidencia unanimidad en que la universidad debe apoyar la innovación en diferentes sectores productivos y sociales con sus conocimientos.

Figura 6. Respuestas de docentes e investigadores con respecto a la responsabilidad de la universidad en apoyar la innovación de diferentes sectores productivos y sociales con sus conocimientos

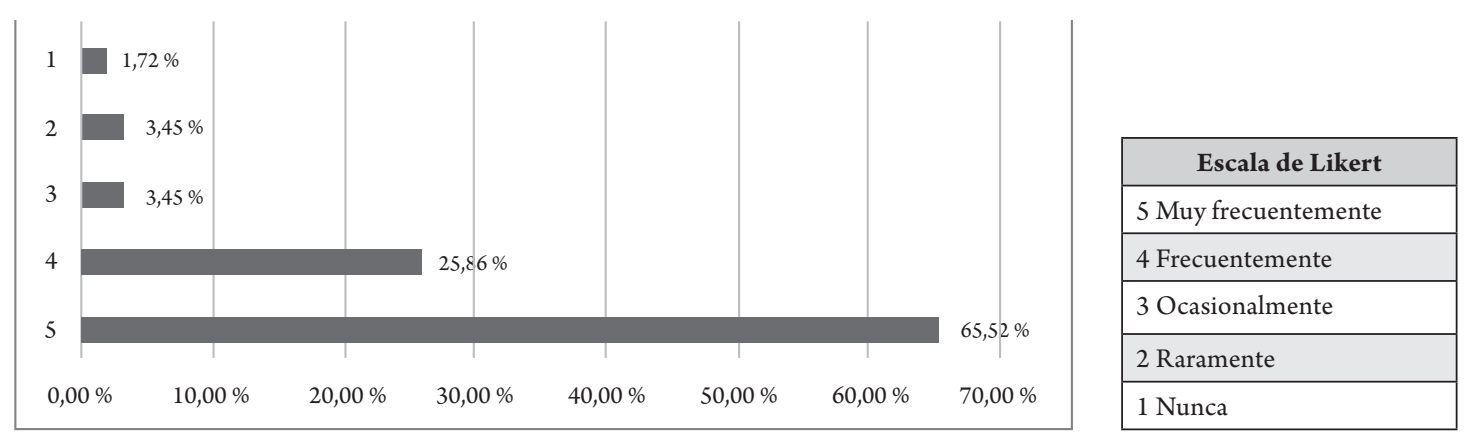

Fuente. Elaboración propia.

De los encuestados, un 51,7 \% considera ocasional el apoyo a los docentes e investigadores para desarrollar y consolidar organizaciones tipo spin-off/spin-out como resultado de sus proyectos o áreas de conocimiento. 


\subsubsection{Conocimiento de tecnologías y plataformas.}

Un 67,2 \% no conoce la existencia de estrategias de crowdsourcing para el desarrollo de proyectos, lo cual se presenta como un reto para la facilitación del desarrollo de los procesos de innovación abierta. El mismo porcentaje de docentes e investigadores desconoce la existencia de propiedad intelectual conjunta o cruzada. En el caso de la financiación, el $81,8 \%$ desconoce la existencia de modelos de crowdfunding en la realización de proyectos.

Se encuentran opiniones divididas con respecto a la existencia de plataformas o portales que permiten divulgar los conocimientos creados en la Universidad Distrital, resultado de la academia, la investigación y la extensión. Para facilitar el planteamiento de soluciones a problemas que generen un alto impacto en la sociedad, solo un $36 \%$ conoce la existencia de mecanismos que permiten a la universidad conocer las necesidades del entorno público o privado para resolver una necesidad particular.

Con una visión de reflexión se encuentra que un $75,8 \%$ de los encuestados desconoce propuestas de innovación abierta en las que participa la Universidad Distrital; alineadamente con esto, los resultados evidencian que solo un $15,5 \%$ conoce la existencia de spin-offs en la universidad.

Acorde a la cultura de la universidad, un 91,3\% de los docentes e investigadores encuestados considera importante la existencia de redes de investigación e innovación creadas por la universidad. Además, están de acuerdo con que es necesario el reconocimiento a docentes, investigadores y estudiantes que participen en procesos de innovación abierta.

De los 58 docentes e investigadores encuestados, 28 consideran que es fácil compartir información, conocimiento y tecnologías con otras instituciones para el desarrollo de proyectos, y 30 consideran lo contrario.
De acuerdo con los resultados obtenidos, se identifican los factores relevantes en los procesos de innovación abierta, desde la relación empresaUniversidad Distrital, en la cual para los dos actores es importante la disposición a innovar, la cooperación y la financiación. Los factores diferenciadores para la empresa son la confianza, la agilidad frente al cambio y la estructura de la organización. Mientras que para la universidad los factores son la financiación, el reconocimiento, la comercialización de resultados y la existencia de una plataforma o mecanismo que permita llevar a cabo innovación abierta.

\subsection{Análisis de correlación}

Se realizó un análisis de correlación entre variables cualitativas en el que se validó la relación entre los factores identificados como importantes para cada uno de los actores estudiados. Estos factores fueron los que se enlistan a continuación.

- Para la empresa. La disposición a hacer innovación abierta, confianza, agilidad frente al cambio, estructura organizacional, cooperación, competitividad y financiación.

- Para la universidad. Financiación, propiedad intelectual, cooperación, plataforma, disposición, reconocimiento y comercialización de resultados.

Mediante la metodología de análisis de las tablas de contingencia, a través de la prueba $\chi^{2}-$ chicuadrado- y definiendo la hipótesis nula Ho- las dos variables son independientes, esta es rechazada para los dos análisis, lo que significa que las variables estudiadas en cada uno de los casos están relacionadas y son altamente importantes en la influencia y disposición de los actores a realizar innovación abierta. 


\section{Discusión de resultados}

T a finalidad de los cuestionarios fue, en primer industrial pyme de Bogotá en sus prácticas de gestión para la innovación y la posible disposición al nuevo paradigma de la innovación abierta. La encuesta realizada está dividida en cuatro secciones generales: información de la empresa, conocimiento sobre la innovación, aspectos relevantes para desarrollar innovaciones en la empresa y la disposición a la innovación abierta, para un total de 35 preguntas.

En busca de analizar la relación empresauniversidad se realizó una encuesta a los docentes de la Universidad Distrital en la que se busca conocer su participación en procesos de innovación abierta. La encuesta se divide en tres partes: experiencia en procesos de innovación abierta, nivel de conocimiento relacionado y tecnologías y plataformas que favorezcan la innovación abierta.
Sin embargo, se identifica que la razón por la cual las pymes desarrollan prácticas limitadas de innovación abierta puede estar asociada al temor de una posible filtración o copia de los recursos estratégicos, perder los beneficios de desarrollar un producto por no tener las capacidades para comercializarlo, no tener el enfoque por demasiada información o no obtener resultados porque es difícil mantener relaciones con otros actores a largo plazo.

Las desventajas mencionadas generan falta de confianza, no solo en las empresas sino en muchos actores, ya que no se percibe una relación estable y hay riesgo de perder o ganar. Así, la confianza es un valor necesario para tener buenas relaciones entre actores; los beneficios de tenerla incluyen el desarrollo de innovaciones de valor, eficiencia en las comunicaciones, responsabilidades y riesgos compartidos, además de un aumento en la competitividad.

\section{Propuesta}

$\mathrm{E}$ xisten diversos actores que pueden participar en los procesos de innovación abierta, como lo son Otras empresas, proveedores, clientes, universidad y Estado, entre otros. Un 84 \% de las empresas encuestadas están dispuestas a trabajar con las universidades como actor principal, validando el objeto de estudio de la presente investigación que se enfoca en los actores universidad-empresa.

A partir de la teoría consultada y los resultados obtenidos en la discusión, se propone como aporte de este artículo el modelo de integración que se presenta en la figura 7 para realizar procesos de innovación abierta entre los actores analizados. 
Figura 7. Modelo de integración InnAUD-empresa propuesto

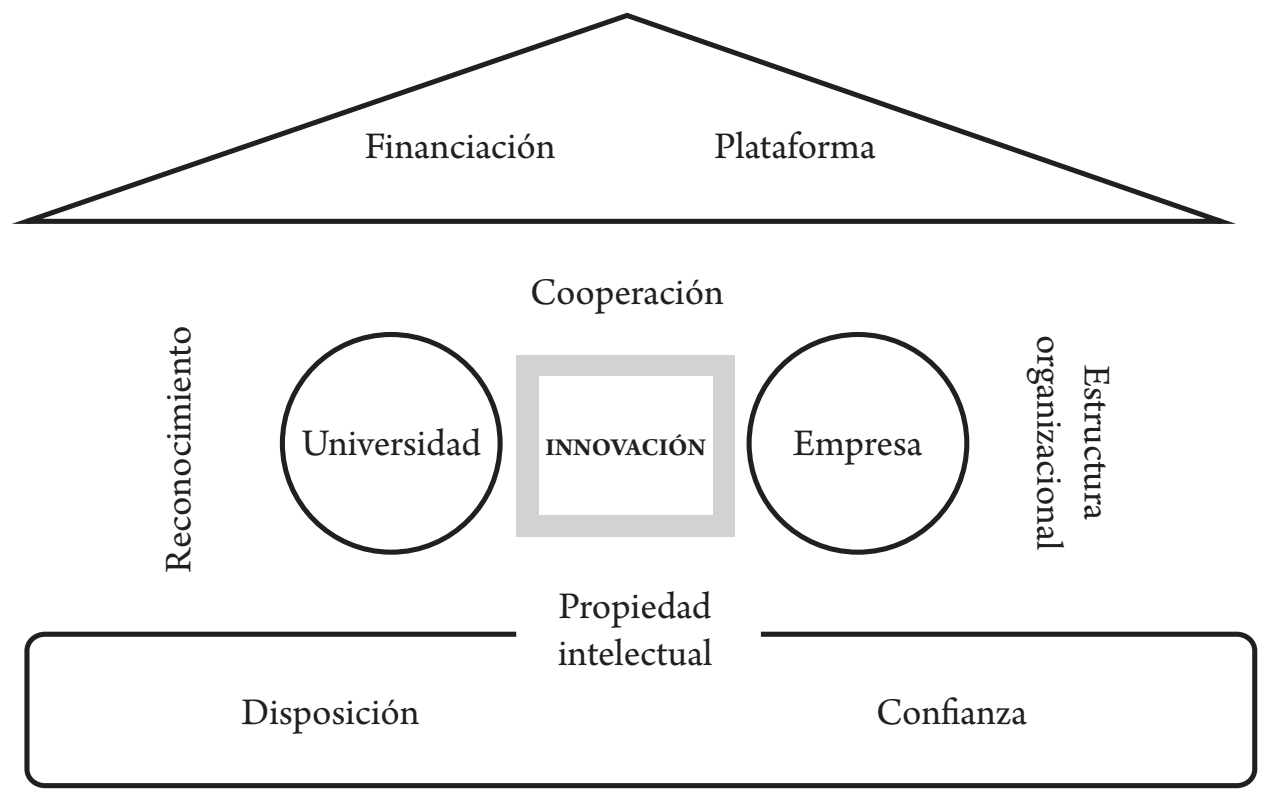

Fuente. Elaboración propia.

El modelo InnAUD-Empresa ilustra los factores correlacionados para cada actor, el cual posee tres partes principales: techo, centro y base. Es importante resaltar que el centro no tiene fronteras, lo cual quiere decir que es un modelo abierto a más factores que puedan intervenir, según el contexto de cada actor participante. Además, se encuentra la palabra innovación, que se convierte en el resultado del proceso.

El rectángulo de la parte inferior hace referencia a las bases que permitirán el desarrollo del proceso de innovación abierta, en este caso la confianza y la disposición de los actores. Posteriormente, se tiene la propiedad intelectual como puente que permite conectar la base con el desarrollo del proceso, en el cual intervienen los actores -universidad, empresa - de forma cooperativa, a través de transferencia tecnológica para la cocreación de nuevas iniciativas $y$, transversalmente, se deben definir estrategias de propiedad intelectual cruzada y licenciamientos.
Para la Universidad Distrital el factor reconocimiento es un condicionante en la realización de procesos de innovación abierta. Por otro lado, para la empresa su estructura organizacional se convierte en un factor fundamental en el propósito de lograr interacciones ágiles y adaptativas al entorno, por lo que es necesario crear los lineamientos o las políticas que exige el proceso de innovación abierta.

Finalmente, se observa que en el triángulo superior o techo del modelo se encuentran los factores facilitadores del proceso, como lo son la existencia de una plataforma — crowdsourcing — o mecanismo de fusión, conexión entre los actores y financiación que puede ser a través de crowdfunding; esta última se presenta como factor facilitador y no base, lo cual quiere decir que no se considera como barrera principal para iniciar procesos de innovación abierta. 


\section{Conclusiones}

T os principales factores identificados, de Jacuerdo con las prácticas de innovación de las pymes manufactureras encuestadas en Bogotá, son confianza, disposición, cooperación, estructura organizacional, financiación y plataforma o mecanismo de conexión. Se evidencia que la financiación no es una barrera para el desarrollo de procesos de innovación y se afirma que la confianza es un factor clave para el buen funcionamiento de estos procesos.

La principal oposición para hacer innovación abierta es el temor de compartir recursos que puedan ser copiados o filtrados por quienes participan, lo cual genera una fuerte barrera de entrada que disminuye el interés en desarrollar este tipo de integración y cocreación.

La mayoría de las empresas encuestadas consideran importante la innovación y están desarrollando diferentes tipos de innovaciones en sus organizaciones. De igual forma, tanto las empresas como los docentes e investigadores de la Universidad Distrital afirman su disposición al desarrollo de procesos de innovación abierta con otros actores; se resalta que un $84 \%$ de las empresas encuestadas está dispuesto a trabajar con las universidades como actor principal.

Aquí, cabe resaltar que en Bogotá, la Universidad Distrital desde hace varios años cuenta con una cultura investigadora muy fuerte, lo cual le permite ser un actor con mayor probabilidad de apertura para el desarrollo de procesos de innovación abierta, argumentado con las respuestas de los docentes e investigadores encuestados. Por lo tanto, se plantea un modelo universidad-empresa debido a que un deber de las universidades es ser el motor de conocimiento de la sociedad, por lo que pueden aportar al desarrollo de procesos de innovación abierta junto con el sector empresarial $\mathrm{u}$ otros actores.
El modelo de integración propuesto se presenta como un marco de trabajo, mas no como una metodología a seguir, pues se reconoce la diversidad de posibles entornos y contextos existentes. Este modelo busca resaltar los factores relevantes para los actores en estudio, con el fin de que, basados en este, sea posible definir claramente las metodologías, los acuerdos y las plataformas a utilizar entre las partes, garantizando la relevancia de los factores identificados y asegurando que no hay límite entre ellos en los procesos de innovación abierta.

Para futuras investigaciones se podría profundizar en responder la pregunta: ¿cómo se podría aumentar la confianza de los actores en procesos de innovación abierta? Igualmente, se podría trabajar sobre la definición de beneficios específicos en el desarrollo de procesos de innovación abierta para pymes de forma sectorizada según la actividad económica en Colombia.

\section{Referencias}

Asociación Nacional de Instituciones Financieras. (2020). Gran encuesta pyme. Primer Semestre 2020. Recuperado de https:// bit.ly/3gQzCHs

Barroso, J. M.; Cabero, J. (2013). La utilización del juicio de experto para la evaluación de TIC: el coeficiente de competencia experta. Bordón. Revista de Pedagogía, 65(2), 25-38. DOI: https://doi.org/https://doi.org/10.13042/ brp.2013.65202

Becker, B. A.; Eube, C. (2018). Open innovation concept: integrating universities and business in digital age. Journal of Open Innovation: Technology, Market, and Complexity, 4(1), 12. DOI: https://doi.org/10.1186/s40852-018-0091-6

Bernal-Torres, C. A.; Frost-González, S. (2015). Innovación abierta en empresas colombianas: reto a superar. Revista Venezolana de Gerencia, 20(70), 252-267. DOI: https://doi. org/https://doi.org/10.31876/revista.v20i70.19996 
Cabrera-Flores, M.; López-Leyva, J.; Peris-Ortiz, M.; OrozcoMoreno, A.; Francisco-Sánchez, J.; Meza-Arballo, O. (2020). A framework of penta-helix model to improve the sustainable competitiveness of the wine industry in Baja California based on innovative natural resource management. E3S Web of Conferences, 167, 06005. DOI: https://doi.org/https://doi. org/10.1051/e3sconf/202016706005

Castellar, E. (2020). Una mirada al estado de la educación superior con relación a los Objetivos de Desarrollo Sostenible. Educación Superior y Sociedad, 32(2), 15-35. Recuperado de https://bit.ly/3gVcp7f

Chesbrough, H. W. (2003). Open innovation: the new imperative for creating and profiting from technology. Boston: Harvard Business Press. Recuperado de https://n9.cl/3kdv

Colciencias. (2021). Oficinas de Transferencia de Resultados de Investigación-OTRIS. Recuperado de https://bit.ly/3zVclM7

Crilly, M.; Vemury, C. M.; Humphrey, R.; Rodríguez, S.; Crosbie, T.; Johnson, K.; Wilson, A.; Heidrich, O. (2020). Common language of sustainability for built environment professionals. The quintuple helix model for higher education. Energies, 13(22), 5860. DOI: https://doi.org/10.3390/en13225860

de las Heras-Rosas, C.; Herrera, J. (2021). Research trends in open innovation and the role of the university. Journal of Open Innovation: Technology, Market, and Complexity, 7(1), 29. DOI: https://doi.org/10.3390/joitmc7010029

Departamento Administrativo Nacional de Estadística. (2019). Encuesta de desarrollo e innovación tecnológica industria manufacturera-EDIT IX 2017'2018. Boletín Técnico. Recuperado de https://cutt.ly/LlFT68z

Ecopetrol. (2019). Innovación abierta. Recuperado de https:// cutt.ly/rlFEo0Z

Etzkowitz, H. (2003). Innovation in innovation: the triple helix of university-industry-government relations. Social Science Information, 42(3), 293-337. DOI: https://doi.org/https:// doi.org/10.1177/05390184030423002

Flor, M.L.; Blasco-Díaz,J.L.; Lara-Ortiz, M.L. (2020). Innovation policy instruments through the lens of open innovation. An analysis in the Spanish context. Journal of Evolutionary Studies in Business, 5(1), 52-80. DOI: https://doi.org/https://doi. org/10.1344/jesb2020.1.j068

García-González, A.; Ramírez-Montoya, M. S. (2019). Higher education for social entrepreneurship in the quadruple helix framework: co-construction in open innovation. En M. A. Conde-González; F. J. Rodríguez-Sedano; C. FernándezLlamas; F. J. García-Peñalvo (Eds.) Proceedings of the Seventh International Conference on Technological Ecosystems for Enhancing Multiculturality. (925-929). Nueva York: Association for Computing Machinery. DOI: https://doi. org/https://doi.org/10.1145/3362789.3362794

Genuchten, E.; Calderón-González, A.; Mulder, I. (2019). Open innovation strategies for sustainable urban living. Sustainability, 11(12), 3310. DOI: https://doi.org/https:// doi.org/10.3390/su11123310
Gloor, P. A. (2006). Swarm creativity: competitive advantage through collaborative innovation networks. Oxford: Oxford University Press. DOI: https://doi.org/10.1093/ acprof:oso/9780195304121.001.0001

Glukhova, L. V.; Sherstobitova, A. A.; Korneeva, E. N.; Krayneva, R. K. (2020). VUCA-managers training for smart systems: innovative and organizational approach. En V. Uskov; R. Howlett; L. C. Jain (Eds.) Smart education and e-learning 2020. (361-370). Singapur: Springer. DOI: https://doi. org/10.1007/978-981-15-5584-8_31

González-Millán,J.J.; Rodríguez-Díaz, M. T.; González-Millán, O. U. (2019). Factores que inciden en la gestión de conocimiento y la innovación abierta en empresas colombianas. Revista Virtual Universidad Católica del Norte, (58), 116-138. DOI: https://doi.org/https://doi.org/10.35575/rvucn.n58a10

Hasche, N.; Linton, G.; Oberg, C. (2017). Trust in open innovation-the case of a med-tech start-up. European Journal of Innovation Management, 20(1), 31-49. DOI: https://doi. org/10.1108/ejim-10-2015-0111

Huizingh,E.K.(2011). Open innovation: state of the art and future perspectives. Technovation, 31(1), 2-9. DOI: https://doi.org/ https://doi.org/10.1016/j.technovation.2010.10.002

INNpulsa. (2021). Nosotros. Recuperado de https://www. innpulsacolombia.com/nosotros

Jamett, I.; Alvarado, L.; Maturana, S. (2017). Análisis al estado del arte de la innovación abierta: implicaciones prácticas en la ingeniería. Revista Ingeniería de Construcción, 32(2), 73-84. DOI: https://doi.org/http://dx.doi.org/10.4067/S071850732017000200006

Juca-Aulestia, M.; Labanda-Jaramillo, M.; Guaman-Quinche, J.; Coronel-Romero, E.; Chamba-Eras, L.; Jácome-Galarza, L.-R. (2020). Open innovation at university: a systematic literature review. En A. Rocha; H. Adeli; L. P. Reis; S. Costanzo; I. Orovic; F. Moreira (Eds.) Trends and Innovations in Information Systems and Technologies (Vol. 1, 3-14). DOI: https://doi.org/ https://doi.org/10.1007/978-3-030-45688-7_1

Lam, L.; Nguyen, P.; Le, N.; Tran, K. (2021). The relation among organizational culture, knowledge management, and innovation capability: its implication for open innovation. Journal of Open Innovation: Technology, Market, and Complexity, 7(1), 66. DOI: https://doi.org/10.3390/joitmc7010066

Liu, Q.; Du, Q.; Hong, Y.; Fan, W.; Wu, S. (2020). User idea implementation in open innovation communities: evidence from a new product development crowdsourcing community. Information Systems Journal, 30(5), 899-927. DOI: https:// doi.org/10.1111/isj.12286

López-Garzón, M. A.; Vaca-Sánchez, T. I. (2010). Innovación empresarial en pymes colombianas (Tesis de especialización). Colegio de Estudios Superiores de Administración. Bogotá, Colombia. Recuperado de https://bit.ly/3jbxETC 
Muhammad, F.; Ikram, A.; Jafri, S. K.; Naveed, K. (2021). Product innovations through ambidextrous organizational culture with mediating effect of contextual ambidexterity: an empirical study of IT and telecom firms. Journal of Open Innovation: Technology, Market, and Complexity, 7(1), 9. DOI: https://doi.org/10.3390/joitmc7010009

Ovuakporie, O. D.; Pillai, K. G.; Wang, C.; Wei, Y. (2021). Differential moderating effects of strategic and operational reconfiguration on the relationship between open innovation practices and innovation performance. Research Policy, 50(1), 104146. DOI: https://doi.org/https://doi.org/10.1016/j. respol.2020.104146

Palacios, J.; Ramírez-Montoya, M. S.; Cubo-Villalba, J.; Burgos, D. (2018). Maturity model for collaborative innovation: higher education challenge. Ponencia presentada en el 13th Iberian Conference on Information Systems and Technologies (CISTI). Caceres, España, 13-16 de junio.

Payán-Sánchez, B.; Belmonte-Ureña, L.; Plaza-Úbeda, J.; Vazquez-Brust, D.; Yakovleva, N.; Pérez-Valls, M. (2021). Open innovation for sustainability or not: literature reviews of global research trends. Sustainability, 13(3), 1136. DOI: https://doi.org/https://doi.org/10.3390/su13031136

Sabato, N. R.; Botana, J. (1993). La ciencia y la tecnología en el desarrollo futuro de América Latina. Arbor: Ciencia, Pensamiento y Cultura, (575), 21-44.

Shutyak, Y. (2016). Open innovation practice: a case study of university spin-offs. Journal of Entrepreneurship, Management and Innovation, 12(1), 75-90. DOI: https://doi.org/https:// doi.org/10.7341/20161214

Sudiana, K., Sule, E. T., Soemaryani, I., Yunizar, Y. (2020). The development and validation of the penta helix construct. Business: Theory and Practice, 21(1), 136-145. DOI: https:// doi.org/https://doi.org/10.3846/btp.2020.11231

Temiz, S. (2021). Open innovation via crowdsourcing: a digital only hackathon case study from Sweden. Journal of Open Innovation: Technology, Market, and Complexity, 7(1), 39. DOI: https://doi.org/10.3390/joitmc7010039

Valdez-Juárez, L. E.; Castillo-Vergara, M. (2020). Technological capabilities, open innovation, and eco-innovation: dynamic capabilities to increase corporate performance of SMEs. Journal of Open Innovation: Technology, Market, and Complexity, 7(1), 8. DOI: https://doi.org/https://doi.org/10.3390/ joitmc7010008

Vicente-Saez, R.; Gustafsson, R.; Van den Brande, L. (2020). The dawn of an open exploration era: emergent principles and practices of open science and innovation of university research teams in a digital world. Technological Forecasting and Social Change, 156, 120037. DOI: https://doi.org/https:// doi.org/10.1016/j.techfore.2020.120037
Vlajcic, D.; Marzi, G.; Caputo, A.; Dabic, M. (2019). The role of geographical distance on the relationship between cultural intelligence and knowledge transfer. Business Process Management Journal, 25(1), 104-125. DOI: https://doi. org/10.1108/bpmj-05-2017-0129

Wu, A.; Garimella, A.; Subramanyam, R. (2021). Could government measures crowd out grassroots philanthropy? Empirical evidence from an education crowdfunding platform. En The Hawai'i International Conference on System Sciences (HICSS) (Ed.) Proceedings of the 54th Hawaii International Conference on System Sciences. (4138-4147) DOI: https://doi. org/10.24251/hicss.2021.503

Yuan, C. H.; Li, Y.; Vlas, C. O.; Peng, M. W. (2018). Dynamic capabilities, subnational environment, and university technology transfer. Strategic Organization, 16(1), 35-60. DOI: https://doi.org/https://doi. org/10.1177/1476127016667969

Yun, J. J.; Liu, Z. (2019). Micro-and macro-dynamics of open innovation with a quadruple-helix model. Sustainability, 11(12), 3301. DOI: https://doi.org/https://doi. org/10.3390/su11123301

Yun, J.; Zhao, X.; Jung, K.; Yigitcanlar, T. (2020). The culture for open innovation dynamics. Sustainability, 12(12), 5076. DOI: https://doi.org/10.3390/su12125076

Zakharov, P. N.; Posazhennikov, A. A.; Zakharova, Z. A. (2020). Open innovations as a tool of interaction between universities and business structures in the digital economy. En E. G. Popkova; B. Sergi (Eds.) Lecture Notes in Networks and Systems. (Vol. 87, 301-306). DOI: https://doi.org/10.1007/978-3030-29586-8_35

Zobel, A. K.; Lokshin, B.; Hagedoorn, J. (2017). Formal and informal appropriation mechanisms: the role of openness and innovativeness. Technovation, 59, 44-54. DOI: https://doi. org/https://doi.org/10.1016/j.technovation.2016.10.001 